\title{
Local strategies for competitive, effective and secure energy uses: the RENERGY transfer tools and methods
}

\author{
C. Cosmi ${ }^{1}$, J. Dvarioniene ${ }^{2}$, I. Marques ${ }^{3}$, T. Ferrão ${ }^{3}$, I. Bloomfield ${ }^{4}$, \\ K. Brix ${ }^{5}$ \& D. R. Trummer ${ }^{5}$ \\ ${ }^{1}$ Institute of Methodologies for Environmental Analysis, \\ National Research Council of Italy (CNR-IMAA), Italy \\ ${ }^{2}$ Kaunas University of Technology, \\ Institute of Environmental Engineering, Lithuania \\ ${ }^{3}$ Intelligence in Innovation, Innovation Centre, Portugal \\ ${ }^{4}$ Durham County Council Sustainability Team, County Hall, UK \\ ${ }^{5}$ Slagelse Municipality, Center for Technique and Environment, Denmark
}

\begin{abstract}
The INTERREG IVC RENERGY project aims at contributing to the achievement of the Europe 2020 targets and improving the effectiveness of local sustainable energy strategies by demonstrating the relevance of a holistic approach to tackle local community needs, cultural and infrastructural characteristics. A "three pillar" approach is used to maximize knowledge transfer and results uptake through synergic actions. Case studies and Energy Labs represent the key building blocks of a structured interregional cooperation contributing to the achievement of the project objectives and ensuring continuous dialogue and cooperation between experts, producers/suppliers and end-users. A selection of good practices will be transferred into the Local Implementation Plans including key policy recommendations to be disseminated to target stakeholders groups.
\end{abstract}

Keywords: local sustainable energy strategies, policy making, RES Market Uptake, community involvement. 


\section{Introduction}

The European policy strategy is focused on key issues linked to the main challenges that the communities are facing with: a secure and affordable energy supply, climate change mitigation and social cohesion (COM (2010) 2020 final, [1]) $(\mathrm{COM} / 2010 / 639$ [2]). Moreover, the European Union covers a wide territory where member countries present strong differences in terms of consumption profiles, policy, culture and infrastructures. Citizens' behavior can deeply influence energy and emissions trends with important consequences in terms of environmental quality. As well as local vulnerability to climate change events. Local communities can therefore contribute considerably to the achievement of national objectives and international commitments, playing a leading role in the transition pathway towards a low carbon economy.

In this framework, interregional cooperation is fundamental to promote a "smart, sustainable and inclusive growth" by facilitating and exchange of experience knowledge transfer and by mobilising communities that can work together to achieve the targets and to improve their capabilities.

Many initiatives have been therefore carried out at European level to promote a steady commitment of local authorities on energy and climate issues among which it is worth mentioning Energy Cities [3], the European Association of local authorities promoting energy efficiency, renewable energy and distributed generation; CONCERTO [4], the initiative launched by the European Commission to support local communities in developing and demonstrating concrete strategies and actions that are both sustainable and highly energy efficient; Covenant of Mayors [5], a commitment by signatory towns and cities to go beyond the objectives of EU energy policy in terms of reduction in $\mathrm{CO}_{2}$ emissions through enhanced energy efficiency and cleaner energy production and use; Smart Cities initiatives [6] aimed to demonstrate that the citizens' quality of life and local economies can be improved through investments in energy efficiency and reduction of carbon emissions.

Besides the voluntarily commitment initiatives, sustainable integrated economic development and social cohesion of European regions, regional competitiveness and employment, European territorial cooperation are supported by the European Regional Development Fund [7] that focuses on key issues as energy, environment, risk prevention and innovation.

The INTERREG IVC project "Regional Strategies for Energy Conscious Communities - RENERGY" [8] funded under the 4th Call for proposals of the INTERREG IVC Programme, leaded by the Potenza Province (Italy), involves 12 partners located in 10 European regions (Austria, Denmark, Germany, Hungary, Italy, Lithuania, Portugal, Romania and United Kingdom) among which three knowledge institutions. It focuses on the development of transferable and customizable methodologies, tools and guidelines to be used by the partner's countries for the design and implementation of action plans and sustainable energy policies. Three main dimensions are considered: Policy Making, RES Market Uptake and Community Involvement that constitute the three "project pillars" and are strictly interrelated. The ultimate objective is to improve the effectiveness of 
local sustainability energy policies in response to the EU policies (COM/2010/639 [9]) demonstrating the relevance of an integrative bottom up stakeholder involvement process in the achievement of strategic objectives.

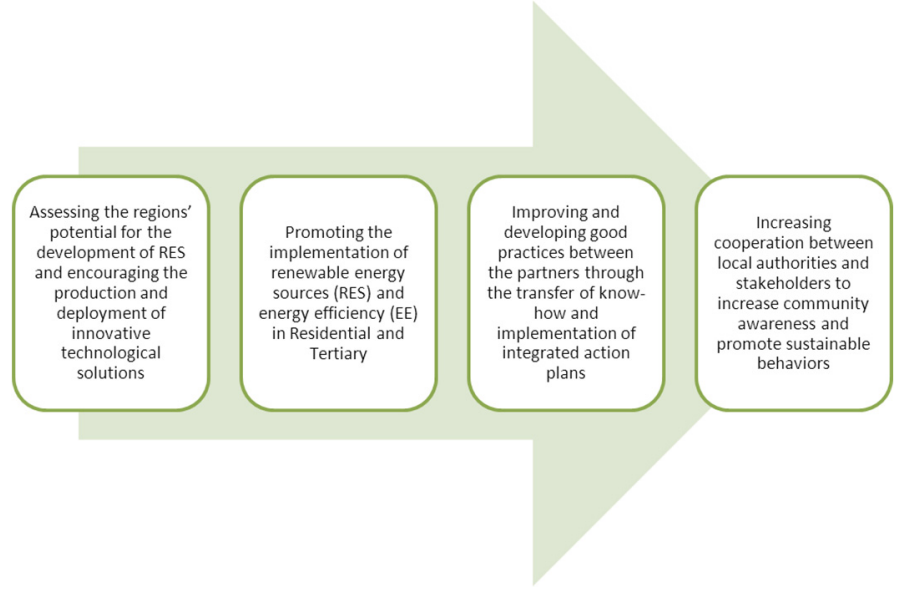

Figure 1: RENERGY operating objectives.

The methodological framework as well as the main results are described extensively in the following.

\section{Methodology}

The 'three pillar regional Energy Labs approach' constitutes the core of the RENERGY methodology that is aimed at maximizing knowledge transfer and tangible results. Knowledge is built through case studies and Energy Labs that guarantee support to the pillars by bringing exchange of good practices further towards local/regional implementation. The essential steps of the project can be resumed in three phases: Build up, Discuss and Share the knowledge. Stakeholders' involvement is foreseen in each of these three phases to provide policy makers with feedbacks and to foster the actuation of policies and measures.

\subsection{The self-assessment analysis: build up the knowledge}

The first step of the project concerns with the evaluation of the national and local policy framework, market conditions, energy infrastructures, renewable and energy efficiency potentials, geographical features, environmental problems, community behaviour, in order to identify the key issues to be enhanced in the local implementation plans. A structured methodology base on the integration of three renowned methodologies (ALEP - Advanced Local Energy Planning, SWOT - Strengths, Weaknesses, Opportunities, and Threats, PEST - Political, Economic, Social and Technological) was set up for the background analysis of 
the partners communities (RENERGY [10], Dvarioniene et al. [11]). The ALEP Advanced Local Energy Planning, developed in the framework of the IEA - ECBCS Annex 33 [13], is aimed at providing an analytical representation of energy systems as a sound basis for energy-environmental planning. The SWOT [14] is applied to a qualitative evaluation of energy systems and widely used as a decision making support tool whereas the PEST [15] is typically utilised in strategic decisions. The integration of these approaches allows a quantitative and qualitative characterisation of local systems that integrates the three dimensions into a unique framework. Data collection was carried out by checklists (top-down approach) to analyse strategic and planning documents, legislation and collecting the statistical information and thematic questionnaires (bottom-up approach) highlighting the social and economic components and integrating statistical information. Three main stakeholder categories were inquired: Local Authorities, Distributors and Grid Operators, Community Members (including citizens, local associations and NGOs). In this way the survey results reflect a common vision of reality by an active involvement of stakeholders.

\subsection{Share the knowledge: the case studies exchange methodology}

The Case Studies exchange is aimed at promoting RES and EE implementation at local level by a transfer of "best examples" between the partners country. Three exemplar Case Studies linked to the project pillars were set to be replicated as a whole or in some peculiar aspects (e.g. policies, instruments etc.) in the partner countries. The case study exchanges examine the main factors that can support their transferability (e.g. attractiveness, competitiveness, sustainability, replicability, compliance with EU Directives, etc.) and the necessary conditions for their transferability (relevant factors influencing/hampering its transferability as policy, strategy, financing. awareness or activity of community) as well as the envisaged results and/or positive side effects deriving from the good practice implementation and the benefits that can derive from its implementation. On site case study exchange visits are also organised to allow interested people to obtain direct information about the implementation of relevant good practices.

Besides the three exemplar case studies, in each of the partner communities a number of good practices contributing significantly to the project themes were selected and collected in a Good Practice Guidebook [16].

\subsection{The stakeholders involvement: the Energy Labs concept}

Stakeholders involvement is aimed at informing and involving actively the communities to discuss needs and opportunities as well as managing possible conflicts and oppositions in the framework a participative and democratic planning and decisional process. Stakeholders commitment in fact allows enhancing their perception of energy and environmental issues, supporting community acceptance of policy strategies and ensuring their operating implementation. The methodology adopted in the RENERGY project (Dvarioniene et al. [12], [17]) is based on the application of the "Living Lab" concept (Cosgravea et al. [18], García-Guzmán et al. [19]) to policy making. Living labs are user-centered, open- 
innovation ecosystems, often operating in a territorial context (e.g. city, agglomeration, and region), integrating concurrent research and innovation processes within a public-private-people partnership. The Energy Labs work out this concept to build up a structured platform aimed at ensuring a close cooperation between energy experts, producers/suppliers and local authorities encouraging and coordinating stakeholder engagement. Stakeholder selection and the identification of the appropriate procedures for their involvement are the core of Energy Lab working methodology. A broader and active participation of all the groups of interest should be guaranteed in order to maximize their contribution to the policy making process. The analysis and selection of the possible stakeholders strongly depend on the specific purpose of the Energy Lab: In particular, their role should be defined carefully in order to identify a supportive and motivated stakeholder set. In general, stakeholders should represent all the relevant categories of community and government, including political representatives, national and regional lawmakers, representatives of financial institutions and the business community, thematic and technical experts, local community members (community leaders, representatives of civic groups, etc.) and NGOs. The selection of stakeholders should consider their motivation as well as the advantages and the disadvantages that can derive from their involvement in the decisional process.

After the identification of the main categories and their representatives it is necessary to define a clear frame for their successful commitment and agree on the rules of participation (for instance which decisions can be influenced by stakeholders) to delineate and understand each party's role. Different kind of events are envisaged to maximise stakeholder participation and to elicit their consensus depending on the issues to be discussed/communicated and the stakeholders involved in the consultation process (e.g. fairs, festivals, thematic workshops, conferences, seminars, interactive laboratories).

In the RENERGY project three rounds of Energy labs were scheduled according to the project phases. The first round (autumn 2012) is aimed at identifying gaps and needs at regional level (build up the knowledge). The second round (autumn 2013) is aimed at discussing the case studies (discuss the knowledge). The third round (spring-summer 2014) is aimed at maximizing knowledge transfer for the regional implementation plans and draw policy recommendations (share the knowledge).

\subsection{The policy strategies: the Model Implementation Plan}

The Model Implementation Plan should outline the vision, objectives and key actions for a public service based on its regional development dynamics, strengths and natural resources, and provide the opportunity for Energy Conscious Sustainable Communities to take advantage of innovative RES and EE measures to provide an effective and efficient environment in which citizens, communities and businesses can flourish. When developing the Plan, it is important to emphasise the role of local and regional governance bodies and appropriate RES and EE polices. Key objectives should demonstrate the relevance of a holistic, integrative, bottom up approach to take local community needs, demands, cultural and infrastructural characteristics into consideration. The three pillar approach 
(Policy Making, RES Market Uptake and Community Involvement) should demonstrate the benefits of the Energy Labs and case study exchanges and establish how the public service will engage with the community in facilitating this integrative, bottom up process. Energy Lab experiences should express an understanding of local issues and focus actions upon specific identifiable needs with measurable RES \& EE outcomes. Additionally, case study exchanges should be developed to promote collected good practice opportunities and support the transfer of knowledge in domestic, public and commercial sectors. A suitable Implementation Plan should include:

- The vision, which would suggest an overarching, long term, normative view on the future and how policy makers see the development of their region.

- Key objectives, which identify some specific, measureable results that regions want to achieve in order to realise their vision.

- A Strategy overview, that will identify the starting point of the Implementation Plan and main focus themes that characterise all the strategic measures and relevant actions in the plan.

- The identification of Intervention fields, which describe the topics covered by the action plan in relation to the project framework.

- Specific actions, which are structured in three strategic lines:

- $\quad$ Community engagement

- Market uptake

- $\quad$ Policy development

\section{Results and discussion}

The results describe the application of the methodology in the partner countries in the first two phases of the project.

Self-assessment analysis of local communities is a basic step to build up the knowledge as it allows highlighting the key issues for the development of the local energy strategies and defining feasible sustainability objectives. Although the deep geographical and socio-economic conditions of the communities that influence obviously energy consumption the self-assessment analysis, addressed at characterising the 11 partners communities respect to the project themes, contributed to emphasize the common issues to be exploited to support an effective knowledge transfer (RENERGY [20]).

As concerns policy making all the countries except Hungary have defined a national energy strategy and most of the communities have also defined regional and local strategies in line with them, to achieve or exceed the 2020 European goals. In fact, most of the communities are signatories Covenant of Mayors whereas Worms and Tulln are also members of Climate Alliance. A composite system of financial mechanisms, such as fiscal incentives, taxation, subsidies to the investment, feed - in tariffs and quota systems supported RES and EE and promoted new businesses opportunities in the different EU countries. As concerns the market characterization the examined regions present a composite economic structure. Unemployment rates are in line with the average EU value (about 10\%) spanning from $4 \%$ of Sheffield (UK) to $12 \%$ of Jaslo (PL) in 2011. Electricity and 
gas prices as well as energy tax regime differ significantly between the countries, influencing the deployment of more efficient technologies. Among the end-use sectors Households and Transport account for the majority of energy consumption in most of the countries, mainly fulfilled by fossil fuels. Industry consumption reflects mostly the industrialization level of the communities and technology efficiency. Commerce and Services contribute greatly to almost all the local economy and can beneficiate by RES and EE exploitation.

As concerns infrastructures the dwelling heating systems and housing stock greatly differ among countries as well as the technology diffusion. RES technologies, except wood fired boilers, are not widespread. Solar and wind are the most accepted, followed by geothermal and biomass.

All the communities support the development of RES and EE, although RES technologies installation is considered too expensive. Uncertainty about the payback time represents a major market barrier together with a scarce knowledge of citizens about their energy consumptions and the potential benefits deriving from technology innovation. Also the level of awareness and concerns towards energy and environmental problems is very different therefore community involvement methods and measures differ a lot among the partners' regions, strongly depending on their policy objectives and culture. It can be seen that except Szentes all the communities are involved in many initiatives to promote the awareness towards RES and EE issues. In particular, Torre Vedras (PT) is the most active whereas Slagelse (DK) has a proven and long tradition in the involvement of stakeholders in municipal activities.

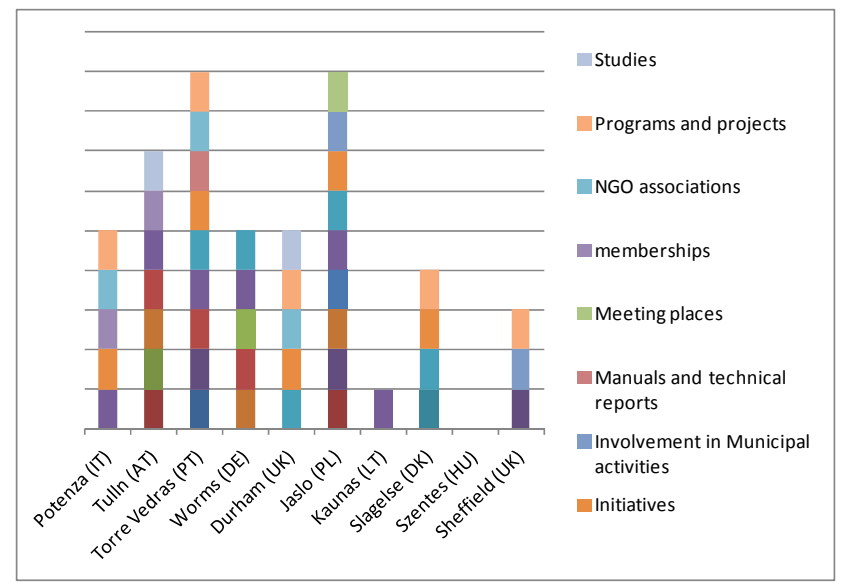

Figure 2: Participation of RENERGY partners in RES and EE initiatives.

As concerns the transfer of knowledge and the increase of the skills and competences, the three case studies addressed complementary problems and issues in the development of local sustainability strategies. The three examples were developed by the Potenza Province (Policy Making) [21], and the municipalities of Tulln (Market Uptake) [22] and Slagelse (Community Involvement) [23]. 
The Potenza Province is a Supporting Structure' for the Covenant of Mayors whose mission is to provide a strategic guidance and technical support to the signatories of Covenant of Mayors. The case study "Living Basilicata - from coordinated sustainable policies to sustainable integrated actions" is therefore focused on the definition of a strategic policy framework to promote RES and EE solutions at local level. The operating objectives are: implementing RES and EE solutions into the public buildings owned by the Province; spreading a "sustainability" culture to improve citizens' behaviors; promoting the use environmental management systems on the provincial territory. In this framework, project experiences and good practices mainly inherent energy efficiency in the building sector were shared with partners. The main factors supporting its transferability are that an integrated policy framework to promote EE and RES that, together with a high political commitment can endorse sound development opportunities for local communities and territories. Moreover, the economic revenues arising from energy saving for public administrations can be re-invested in RES and EE with a multiplying effect.

Concerning energy and RES use, Tulln is leading the way in the region Lower Austria and acts as a multiplier for suitable and feasible technologies as well as for new management and financing concepts in the field of renewable energy use and energy savings. The Tulln case study shows the operative implementation of innovative examples of RES plants that can be considered best examples for other regions. Furthermore, the Tulln case study provides detailed information for the commercial run of a RES system, from the initial idea to invest in alternative energy to the operating implementation. The message that this case study intends to convey is that the investments on RES plants have a sound future perspective as sustainable technologies can preserve the nature and the environment for the future generations

Slagelse case study focus on the set up of a network of energy responsible personnel to achieve higher energy saving targets and improve energy saving behaviour. Slagelse has a tradition in energy renovation of municipal buildings and many of the municipal buildings are equipped with SCADA systems that automatically measure energy and water consumption. Moreover, there is one person appointed responsible for energy consumption in all buildings. The overall objective of the case study is a further reduction of energy consumption and thereby a reduction of $\mathrm{CO}_{2}$ emission from municipal operation - and knowledge sharing between people working in the same field of action. The main factors that support the transferability of this case study are the political wish that municipality could serve as a good example, a political objective for energy reduction in the form of a goal, an action plan and a budget for energy renovations. The envisaged results and/or positive side effects deriving from the good practice implementation are controlled energy consumption and a forum of knowledge sharing.

After the case study visits and an in depth good practices analysis, each partner community has been developing its Local Implementation Plans on the basis of the Model Implementation Plan. Each plan must include one or more good practices (as a whole or a part) to maximize the transfer of knowledge and to derive effective policy recommendations based on the mutual experience and learning. 


\section{Conclusions}

Sustainability is a global challenge that requires an integrated approach to tackle all the components and work out robust solutions and definite energy technology roadmaps. A novel political vision that integrates policy and governance, business development and education involving actively the main stakeholders can therefore encourage the transition towards a green economy. In this framework interregional cooperation represent a strategic tool to disseminate exemplar best practices and to reinforce the community role and consciousness allowing to compare different experiences, motivations and aspirations of the communities and to overcome by joint efforts the main common barriers for the implementation of sustainable energy strategies.

\section{Acknowledgements}

This research was carried out in the framework of the Regional Initiative Project Regional Strategies for Energy Conscious Communities RENERGY (Project no: 1245R4; Duration: 01/01/2012-31/12/2014) funded under the 4th Call for proposals of the INTERREG IVC Programme (Priority: Environment and risk prevention; Theme: Energy and sustainable transport). More information is available at: http://www.renergyproject.eu

\section{References}

[1] Europe 2020: a strategy for European Union growth [COM (2010) 2020 final]

[2] Energy 2020 - A strategy for competitive, sustainable and secure energy [COM/2010/639]

[3] Energy Cities, http://www.energy-cities.eu/

[4] CONCERTO, http://concerto.eu/concerto/

[5] Covenant of Mayors http://www.covenantofmayors.eu/index_en.html

[6] European Initiative on Smart Cities, http://setis.ec.europa.eu/set-planimplementation/technology-roadmaps/european-initiative-smart-cities

[7] European Regional Development Fund http://ec.europa.eu/regional_ policy/thefunds/regional/index_en.cfm

[8] INTERREG IVC Project Regional Strategies for Energy Conscious Communities www.renergyproject.eu

[9] Energy 2020 - A strategy for competitive, sustainable and secure energy [COM/2010/639]

[10] INTERREG IVC Project Regional Strategies for Energy Conscious Communities - RENERGY Report on "Renergy Methodology: SelfAssessment Analysis - Guidelines for Project Partners, October 2012

[11] Dvarioniene, J., Gurauskiene, I., Gecevicius, G., Cosmi, C., Di Leo, S., Selada, C., Marques, I., Mendes G., A holistic approach to sustainable energy development at regional level: the RENERGY self-assessment methodology 7th International Conference Environmental Engineering and 
Management - Integration Challenges for Sustainability - Section VII Waste Management for Resources and Energy Recovery, pp. 303-304, 2013

[12] Dvarioniene, J., Trummer, D. R., Gurauskiene, I., Gecevicius, G., Selada, C., Marques, I., Cosmi, C., "Energy Lab" and stakeholders' involvement as a key tool for energy conscious communities International conference "Ecological Architecture" pp. 18-19, 24, 2013

[13] International energy agency. Advanced Local Energy Planning (ALEP). A Guidebook. Karlsruhe, Germany, 2000; 1: 203

[14] Terrados, J.; Almonacid, G.; Hontoria, L. Regional energy planning through SWOT analysis and strategic planning tools: Impact on renewables development. Renewable and Sustainable Energy Reviews 2007; 11-6: 1275-1287

[15] SWOT Analysis Vs PEST Analysis and When to Use Them http://creately.com/blog/diagrams/swot-analysis-vs-pest-analysis/, 2012

[16] INTERREG IVC Project Regional Strategies for Energy Conscious Communities - RENERGY Report on "Good Practices Guidebook", December 2013

[17] INTERREG IVC Project Regional Strategies for Energy Conscious Communities - RENERGY Report on "Guidelines for stakeholder involvement and Energy Lab methodology", October 2012

[18] Cosgravea, E., Arbuthnot, K., Tryfonas, T. 2013. Living Labs, Innovation Districts and Information Marketplaces: A Systems Approach for Smart Cities. Proceedings of Conference on Systems Engineering Research 16 (2013), 668-677

[19] García-Guzmán, J., Alvaro Carpio, F., Amescua, A., Velasco, M., 2013. A process reference model for managing living labs for ICT innovation: A proposal based on ISO/IEC 15504. Computer Standards \& Interfaces 36(1), 33-41

[20] INTERREG IVC Project Regional Strategies for Energy Conscious Communities - RENERGY "Self Assessment Synthesis Report", September 2013

[21] INTERREG IVC Project Regional Strategies for Energy Conscious Communities - RENERGY "Case Study Concept: "Abitare Basilicata" from coordinated sustainable policies to sustainable integrated actions" TG1: Policy Making, June 2013

[22] INTERREG IVC Project Regional Strategies for Energy Conscious Communities - RENERGY "Case Study Concept: City of Tulln" TG2: Market Uptake, June 2013

[23] INTERREG IVC Project Regional Strategies for Energy Conscious Communities - RENERGY "Case Study Concept: Network of energy responsible personnel” TG3: Community involvement, June 2013 